\title{
EFECTOS DE SENTIDO EN LA ENSEÑANZA DE LENGUAS EXTRANJERAS: UN GESTO DE LECTURA
}

\author{
Gladys BeatrizMorales ${ }^{1}$
}

\section{Resumen}

El artículo tiene por objetivo trazar un relato de la enseñanza de lenguas extranjeras (ELE) en Argentina, comprendiendo el período entre 1950 y la actualidad. Para esto, se buscó poner en evidencia la creciente complejidad que impregna a esta enseñanza con el avance de nuevas teorías. A partir de una triangulación teórica entre programas de investigación de la enseñanza, tendencias teóricas de formación docente inicial y abordajes metodológicos para la ELE, se constatan tres efectos de sentido: el efecto tecnocrático, el de especificidad y el de complejidad. Se considera que la perspectiva que consiste en reducir la complejidad a un conjunto de aspectos aislados se abandona paulatinamente, llegando al enfoque pedagógico predominante en la actualidad que ilustra las interacciones entre la cultura en la cual se inserta la ELE y las condiciones de superación del marco estrecho de esta cultura para aprender con la alteridad.

Palabras clave: Enseñanza de Lenguas Extranjeras; Investigación de la Enseñanza; Formación Docente; Efectos de Sentido

\footnotetext{
${ }^{1}$ Profesora Asociada de la Universidad Nacional de Río Cuarto (UNRC), Argentina. Doctora en Educación (UFRGS-Brasil). Profesora Superior de Francés y Profesora de Portugués. Dirección: Ruta 8 km. 306, Campus Universidad, 5800 - Argentina, Telefone: (0358) 4676579.Email: electrónico:gmorales@ hum.unrc.edu.ar
} 


\section{INTRODUCCIÓN}

La enseñanza de una lengua extranjera $(E L E)^{2}$, como toda prácticade enseñanzatiene una historia y un devenir que la va complejizando. A los primeros vestigios de didáctica de Lengua Extranjera (DLE), sostenida básicamente en la denominada metodología de la enseñanza de lenguas extranjeras, se fueron sumando nuevas miradas, nuevas conjeturas o supuestos que se confirmaron a modo de teorías, se fueron sumando nuevos acontecimientos históricos que complejizaban la mirada del objeto de estudio y en este caso, de la enseñanza de la lengua extranjera (ELE).

Hablar deELE nos lleva a reflexionar sobre el objeto de estudio de tal enseñanza, sobreconceptos de la didáctica,aspectos epistemológicosque orientan las discusiones en la academia, los docentes, los alumnos...

En la búsqueda de atender esos aspectos, este artículo propone establecer puentes entre programas $^{3}$ de investigación de la enseñanza, enfoques en la didáctica de lenguas extranjeras y modelos de formación docente. Para tender esos puentes, nos ha movilizado el interés de dar visibilidad a algunos aspectos que configuran la complejidad involucrada en la enseñanza de una lengua extranjera.

\section{CONTEXTO DEL ESTUDIO}

\subsection{La complejidad objeto de estudio en la enseñanza de lengua extranjera}

En Argentina, la asignatura "Investigación educativa" es inevitable en las curriculas de formación de grado de profesores de Lengua extranjera. El sentido de esa disciplina es claro: que los alumnos (futuros profesores) conozcan losenfoques epistemológicos del objeto de enseñanza (la lengua extranjera), los programas de investigación que orientan el desarrollo del

\footnotetext{
${ }^{2}$ En el artículo nos referiremos más a Enseñanza ya que pretendemos hacer referencia al saber educativo situado en un espacio: la escuela, el aula de clase. Más específico que el concepto de Didáctica, aunque en algunas ocasiones hagamos referencia a esta última.

${ }^{3}$ El concepto de Programas de investigación es propuesto por Shulman (1989), quien lo toma de Lakatos, para describir los géneros de indagación que se encuentran en el estudio de la enseñanza.
} 
conocimiento de la enseñanza, resaltando las singularidades de la investigación educativa en lenguas extranjeras, los aspectos que caracterizan la práctica pedagógica y la práctica docente en esa enseñanza.

Preguntarse sobre qué tipo de objeto es la lengua, es un punto de partida en la investigación educativa ${ }^{4}$ y va a impregnar la lectura de los diversos enfoques didácticos.Duhalde (2008) considera que el objeto de estudio de la investigación educativa siempre es construido; no podemos hablar de él como un objeto real. Decir que es un objeto construido significa que éstees resultado de la reflexión, del intelecto,que ese objeto llamado lengua es producto deconstrucciones teóricas. Lalengua es un aspecto teórico y conjeturado del fenómeno lingüístico(SAUSSURE, 1945).Así, es completamente diferenciado del objeto real, la cosa.

De esta manera, en laELE debemos apoyarnos en disciplinas o teorías científicas que se ocupan de objetos que no se ofrecen directamente a la experiencia, por ejemplo:la estructura del lenguaje. Cuando hablamos de un "adverbio", de una "frase nominal", de una "secuencia descriptiva",etc, suele sorprender al docente la dificultad de los alumnos para comprender "eso" que estamos enseñando. Sin embargo, "eso" es un objeto construido, una construcción teórica. Preguntamos alos alumnos ¿alguna vez vieron pasar caminando a un adverbio ¿ Entre risas, asombro y reflexión ellos captan la idea de objeto construido y la complejidad del objeto a enseñar cuando sean profesores de LE.

Desde esta posición epistemológica nos ubicamos en un marco de complejidad donde reconocemos que en la ELE se entrecruzan diversas disciplinas provenientes de las ciencias de la educación,las ciencias del lenguaje, las ciencias de la sociedad, la psicología, cada cual aportando elementos para preguntarnos ¿A quién se enseña? ¿Quién enseña?¿Cuándo? Qué enseñar?¿Dónde? Con qué finalidad?¿Con qué materiales enseñar? Qué manuales? Qué tipos de evaluaciones?¿Qué estilos de aprendizajes se involucran? Preguntas que delinean el núcleo de la DLE.

Besse (1989,p.33) traza una excelente topología del ámbito de ésta, permitiéndonos visualizar el entrecruzamiento de disciplinas que configuran las preguntas propias de ese

\footnotetext{
${ }^{4}$ Entendemos a la investigación educativa centrada en lo pedagógico, sea referida a: los estudios históricos sobre la pedagogía, la definición de su espacio intelectual, la investigación aplicada a objetos pedagógicos. Todo en busca del mejoramiento de la educación, como es el caso de la indagación sobre el currículo, los métodos de enseñanza y demás factores inherentes al acto educativo: tiempo de aprendizaje, medios, materiales, organización y clima de la clase, procesos de interacción o comunicación (RESTREPO GOMEZ, 2002).
} 


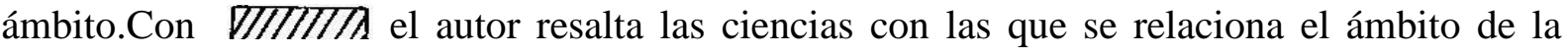
DLE, y nos representa esa complejidad.

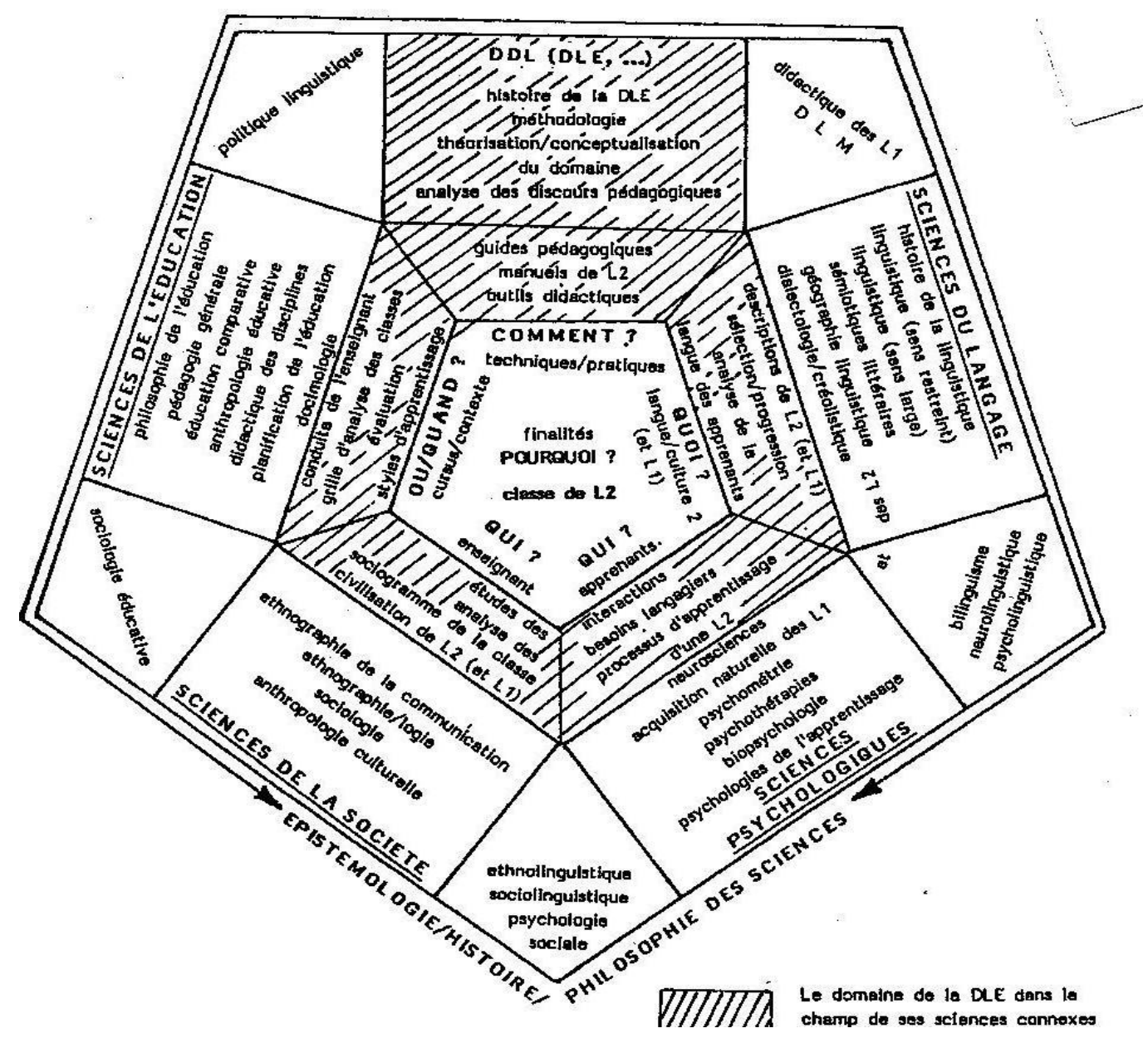

Fig. 1:

Nos situamos, entonces, en un territorio donde la interpretación nos permite indagar los efectos de sentidos ${ }^{5}$ que emergen del gesto ${ }^{6}$ de lectura practicado en esta propuesta,referido a programas de investigación de la enseñanza, tendencias teóricas dela

\footnotetext{
${ }^{5}$ La expresión "efectos de sentidos", es una categoría teórica que singulariza al ámbito del Análisis del Discurso. Este trabajo no se enmarca específicamente en el Análisis del Discurso, pero sí rescata aquel término por la pertinencia con esta propuesta.

${ }^{6}$ SegúnPêcheux (1994, p. 57), leer el archivo, "entendido no sentido amplo de documentos pertinentes e disponíveis sobre umaquestão", involucra el problema de la lecturao, como indica el autor, implica gestos de lectura. No significa ni una lectura puramenteinterpretativista, ni una lectura puramente amarrada a la tradición de la cultura científica. Elgesto de lectura, o sea, la manera de interpretar el archivo, es destacada como práctica que debe ser pensada, discutida.
} 
formación docente inicial ${ }^{7}$ ypropuestas de la DLE. Gesto interpretativo que nos lleva hacia una lectura sensible frente a las "cosas dichas" en las teorías y haceemerger efectos de sentidos diversos, incompletos, reales, variables ${ }^{8}$.

\section{LOS EFECTOS DE SENTIDO}

\subsection{El efecto tecnocrático en la investigación de la enseñanza}

Alrededor de los años 50, en América del Sur y básicamente en Argentina la enseñanza escolar de LE estaba fuertemente marcada por las orientaciones provenientes de Estados Unidos y de Europa. Se hablaba demetodologías de enseñanza, entendidas como posibles caminos para llegar a una determinada meta en la ELE. Esa meta era una enseñanza eficaz de la LE, sentido clásico de la metodología concebida como camino marcado por pasos, técnicas, instrumentos que trazan un recorrido para llegar a la meta: una enseñanza "eficaz".

Cuando decimos "eficaz", nos preguntamos ¿en qué sentido?, el de delimitar con claridad qué acciones, qué caminos debía tomar el docente para lograr el aprendizaje del alumno ${ }^{9}$. Las acciones del docente eran decisivas en la clase. El camino entre las acciones de enseñanza del profesor y el rendimiento del alumno se consideraban a modo de relación lineal entre causa y efecto o, en otras palabras, las "buenas" acciones del docente conducían al "buen rendimiento" de los alumnos.Esta perspectiva de enseñanza era coherente conla tradición de la psicología conductista aplicada y el correspondiente análisis de las tareas y adiestramiento (SHULMAN, 1989).

\footnotetext{
${ }^{7}$ Entendemos a la formación docente inicial como el proceso que tiene como principal finalidad la preparación teórico-metodológica para el ejercicio idóneo de la práctica docente. La diferenciamos de la formación docente continua que refiere a la preparación querealizan los docentes después de graduarse (VOGLIOTTI y MORALES, 1996). En este artículo nos referiremos a la formación docente inicial aunque a veces no la indiquemos de esa manera, sólo para evitar redundancia.

${ }^{8}$ Por una cuestión de delimitación cronológica del marco teórico y de extensión de este trabajo, se dejarán de lado otras líneas de formación docente (como la Academicista),metodologías de la enseñanza de lenguas extranjeras (Tradicional, Natural, Directa), programas de investigación (Tiempo de aprendizaje académico, Cognición del profesor y toma de decisiones) sin perjuicio del análisis que se presenta.

${ }^{9}$ En esa perspectiva, el aprendizaje era entendido como "rendimiento" del alumno (SHULMAN, 1989).
} 
Este género de investigación de la enseñanza se denominainvestigación del procesoproducto, también llamado investigación dela eficacia de la enseñanza. Desde el punto de vista epistemológico se caracterizaba por el positivismo,materializado en la psicología conductista y en una concepción de la práctica pedagógica como acciones lineales entre profesor y alumno.

Indica Shulman (ib., p.25) que el proceso-producto, el día de clase podía ser estudiado siguiendo acciones aisladas como por ejemplo la frecuencia de preguntas difíciles que proponía el docente o como "cadenas de secuencias" de:

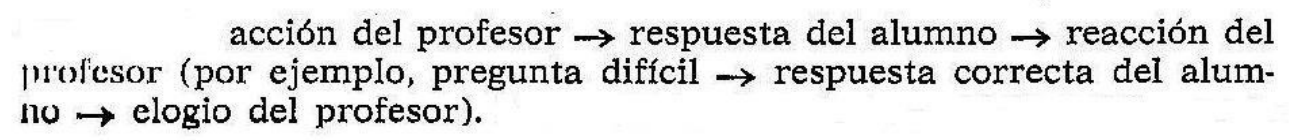

La eficacia de la enseñanza era atribuida a combinaciones de actividades del docente que debían ser discretas y observables, independientes de tiempo y lugar. En la búsqueda de la eficacia se delimitaban criterios que permitían considerar que un docente había realizado las tareas programadas y las correlacionaba con la potencialidad de éstas para alcanzar el logro del criterio de eficacia.

En este enfoque de la investigación de la enseñanza no se diferenciaban los estados cognitivos y afectivos de los alumnos, como tampoco se desarrollaban teorías debido al carácter básicamente descriptivo y correlacional. Así, poco a poco este programa fue perdiendo fuerza intelectual entre los investigadores.

\section{2 ...en la formación docente inicial}

Veamos ahora qué se sostenía en el ámbito de la formación docente inicial mientras imperaba el programa de investigación proceso-producto.

La línea de formación docente denominada “tecnicista"también tuvo sus orígenes teóricos en Estados Unidos, durante el período post-segunda Guerra Mundial. La situación socio-política de Argentina con Estados Unidos, la participación de UNESCO y de la OEA 
favorecieron la presencia de esta perspectiva en Argentina, en los años de la década del ‘60 y sufortalecimiento en los años '70(VOGLIOTTI y MORALES, 1996).

La fundamentación psicológica de este modelo de formación estaba dada por el neoconductismo de Skinner que sostenía la concepción del sujeto de aprendizaje como "producto" de la sociedad.Las consecuencias de los estímulos ambientales a través de un condicionamiento operante que refuerza positiva o negativamente las respuestas de los sujetos, las contingencias de refuerzo debían ser claramente aprendidas, programadas y controladas por el docente.

Como vemos, este plano epistemológico positivista impregna el ideario del modelo tecnicista reforzando el afán de lograr precisión y seguridad. Así, lo esencial para conocer la realidad del hombre reside en puntualizar los aspectos que puedes ser medidos y observados. La norma es la guía de la acción. La denominación de modelo "tecnicista" o "tecnológico" cobra sentido por cuanto la formación se concibe como una tecnología en tanto que conocimiento científico aplicado al hacer y el actuar,dejando de lado el conocer. (ib.)

La formación docente de grado se organiza siguiendo una secuencia lineal que comienza con el conocimiento científico básico, luego el conocimiento aplicado y finalmente el desarrollo de competencias profesionales. De esta manera, la práctica docente,concebida aquella como un conjunto de normas a seguir y técnicas que se deben aplicar, se sitúa al final de la propuesta curricular.

En síntesis, la formación docente implica la preparación de técnicos eficaces para la enseñanza. De este modo, la práctica docente es impregnada de saberes pedagógico-didácticos de carácter prescriptivo.

\section{3 ¿Y en la enseñanza de LE?}

En tiempos de la investigación del proceso-producto y de la formación docente tecnicista, la ELE también estaba impregnada del efecto de sentido tecnocrático. 
A continuación del ataque de Pearl Habor y la entrada en guerra de los norteamericanos, la armada americana lanzó un programa ASTP (Armspecialised training program) que marcó profundamente la metodología enseñanza de $\mathrm{LE}^{10}$.

En dicho programa, la lengua oral era la reina del aprendizaje. Estados Unidos necesitaba formar en poco tiempo un número suficiente de militares que dominaran las lenguas habladas en los futuros teatros de acción. Fueron los inicios del método audio-oral (MAO) llamado también el método de la armada. Sin embargo, este método tuvo muy poca vida, sólo duró dos años ya que no provocó gran interés en el medio didáctico (PUREN, 1995).

En esas condiciones históricas, los lingüistas americanos dejaron la Modern LanguageAssociation, creada en 1883, para fundar la LinguisticSociety of America. Los manuales de estudio fueron editados en japonés, francés, alemán, holandés y ruso. La ELE estaba dirigida por lingüistas.

Fue principalmente la influencia de la Lingüística Aplicada a la ELE que provocó la entrada de la linguiística en la DLE. Esa influencia tomó gran fuerza en la post-guerra, años 60. En ese sentido los especialistas en didáctica hablaban de una era pre-lingüística y de otra lingüística. A este contexto se sumaba la preocupación de Francia por el avance del inglés como lengua de comunicación, desde la finalización de la segunda guerra mundial. Francia tomó la decisión de profundizar y buscar nuevas alternativas de ELE, en vistas de fortalecer el aprendizaje del francés LE como un asunto del Estado. Alternativas que hasta la actualidad nutren a los diversos enfoques didácticos de diversas lenguas extranjeras. Entre ellas, destacamos la metodología audio-visual (MAV) que entrelazaba el soporte sonoro y el visual. La imagen era el pivotpara hacer visible el contenido semántico de las frases, como también para los componentes no lingüísticos del texto: gestos, actitudes.

\footnotetext{
${ }^{10}$ Desde el siglo XIX, los enfoques metodológicos estaban delineados por Alemania y Francia. Se trataba del método de gramática-traducción, también llamado de método tradicional, cuyo objetivo principal era la lectura y la traducción de textos literarios. Este método convivía con otro denominado método natural, cuyo fundamento consistía en considerar que el motor de aprendizaje de una lengua extranjera era la necesidad de la persona de comunicarse superando las barreras culturales. Esa concepción abrió espacio a diversas disciplinas (psicología, sociología, lingüística) en los métodos, organizando paulatinamente el ámbito de la DLE.A inicios del siglo XX, se entendía que para aprender una lengua extranjera no era necesario pasar por la traducción, como tampoco eran tan importantes la gramática y la producción escrita. Este pensamiento constituyó la base del denominado método directo (MD), que priorizaba la expresión oral ya que era equivalente a la expresión escrita. La lengua era considerada como un fenómeno de comunicación oral que solicitaba el estudio de la lengua oral de uso cotidiano. El MD tuvo poca prevalencia en nuestro país, contrariamente a la metodología audio-visual que le sucedería.
} 
En la MAV se trabajaban las cuatro habilidades aunque se prestara más atención al oral buscando atender la comunicación en LE.Se tomaba en cuenta la expresión de los sentimientos, de emociones, expectativas, no considerados en los métodos anteriores.

No obstante, esa metodología privilegiaba las fases de explicación y de sistematización de estructuras gramaticales, la corrección fonética, las relaciones entre las formas lingüísticas. Puren (1989, p.340) indica que el modelo de jerarquía que sostenía la relación entre teoría y práctica consistía en:

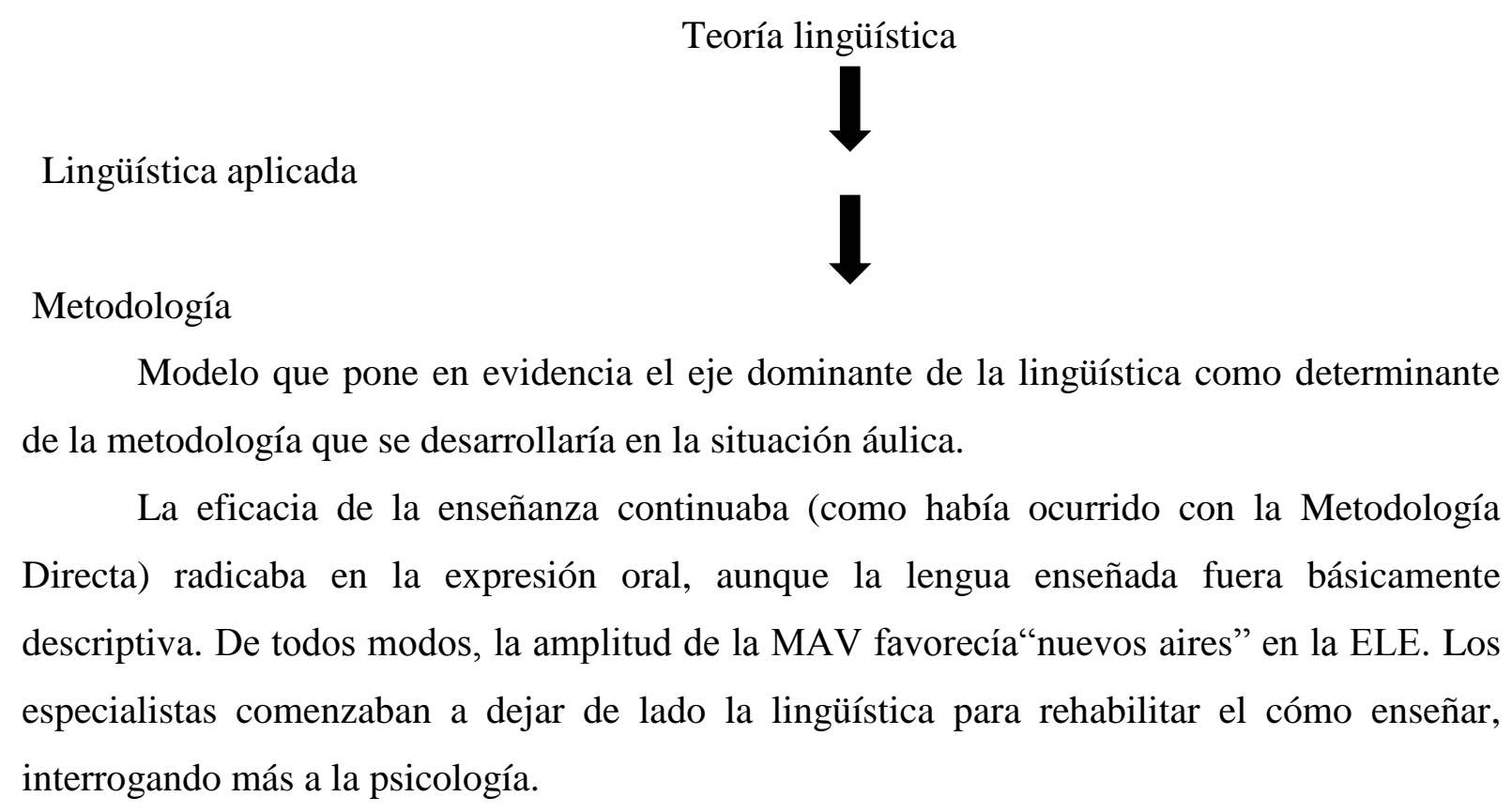

\section{$4 \mathrm{El}$ efecto de especificidad}

\subsection{En la investigación de la enseñanza}

Surge hacia 1960, en Estados Unidos, un programa que se preguntaba ¿Cómo comprenden los alumnos la instrucción que se da en clase?¿Cuáles son los procesos inmediatos y a largo plazo que la enseñanza genera en los alumnos? Se trataba de la investigación de la cognición del alumno y la mediación de la enseñanza, según la cual entre la enseñanza y el aprendizaje median paralelamente procesos sociales (mediación social) y 
procesos cognitivos (mediación cognitiva). Shulman (1989, p.43) ha graficado los proceos de la siguiente manera:

\section{ENSEÑANZA $\rightarrow$ Mediación social $\rightarrow$ Mediación cognitiva $\rightarrow$ APRENDIZAJE}

En la mediación socialse da atención a aquello que el alumno entiende del contexto social en el aula: la manera como se distribuyen los turnos, el carácter de los elogios, de los reproches, las señales que indican las oportunidades de participar, los cambios en las tareas, etc. La mediación cognitiva ocurre concomitantemente con la social del aula, pues se despliegan estrategias metacognitivas, construcciones mentales que pueden ser adecuadas o no para comprender un concepto, un principio, etc.

En la mediación cognitiva toma lugar la psicología cognitiva desde la cual se destierra la concepción de que una tarea cognitivamente simple implica considerar un alumno pasivo que sólo incorpora representaciones verídicas de lo que se le ha enseñado; por el contrario, una tarea cognitiva simple enlaza una tarea activamente cognitiva.

En estas investigaciones el positivismo es debilitado por la perspectiva fenomenológica que da atención a las intenciones, expectativas, construcción de significados de las experiencias de los alumnos. Para comprender por qué los alumnos responden (o no), cómo lo hacen, el docente se pregunta qué comprendieron los alumnos de lo que se les enseñó, dejando de lado la pregunta qué se les enseñó.

El problema no es qué enseñanza es más efectiva, sino qué significados dan el profesor y los alumnos a los hechos de la vida del aula y cuáles son los fundamentos de esas construcciones. La psicología cognitivista y la sociología son las disciplinas de base en estos estudios. También participa la antropología,aunque de manera difusa. Como señala Shulman (ib.,p.44) “el sociólogo y el antropólogo con frecuencia se parecerán, aunque éste último se esforzará más en ver el mundo desde la perspectiva de las categorías fenomenológicas que funcionan para analizar el mundo de los sujetos mismos".

\section{$4.2 . .$. en la formación docente}


Como vemos, estamos incursionando en ideas básicas del cognitivismo, que concibe a la enseñanza como un proceso sociocultural y el conocimiento se logra a través de la identificación, la apropiación y la utilización de la información.

A comienzo de los años '80, en Argentina, la teoría psicogenética de Piaget eramuy estudiada en instituciones de formación de grado de profesores como también en las instancias de formación continua de los educadores.

En la formación docente está implicada una concepción pedagógica que considera a la educación como una construcción humana que involucra necesariamente una interactividad entre sujeto y medio. En este marco, el aprender está vinculado con la apropiación de la información que procede del medio y que el alumno relaciona con otra que ya posee, la reorganiza en sus estructuras mentales, otorgándoles una significación.

Como es muy conocido, Piagetintentó,a través de su teoría, explicar cómo el sujeto conoce la realidad, las etapas, los cambios que se producen en ese proceso de conocimiento. El sujeto incorpora la realidad partiendo de las estructuras cognitivas que posee, asimilando esos contenidos, significándolos, acomodándolos, modificando sus esquemas anteriores proceso que puede afectar de manera leve la estructura cognitiva o que puede modificarla completamente. En ese proceso, el conflicto cognitivo es importante ya que participa directamente en el proceso de construcción de conocimientos. Esto implica que el aprendizaje va a depender del nivel de desarrollo de las estructuras cognitivas del sujeto, considerando que este desarrollo permite programar la secuenciación de contenidos en relación a su complejidad.

La psicología cognitivista de Ausubel $^{11}$ es sumada a la propuesta piagetiana, en la formación docente en Argentina. Ese psicólogo y pedagogo aportó la concepción de que el aprendizaje debe ser significativo para quien aprende y que dicha significatividad está basada en las relaciones entre el conocimiento nuevo y el que ya posee el alumno.

Si bien los estudios de Piaget y de Ausubel tuvieron gran impacto para comprender los procesos de aprendizaje de los alumnos, el lugar del docente sigue vigente, no es apagado, pues en ambos estudios, el alumno es guiado, motivado, interpelado por el enseñante. A éste último le compete la tarea de seleccionar qué enseñar, o sea, la selección de contenidos que

\footnotetext{
${ }^{11}$ No es finalidad de este artículo desarrollar las teorías de Piaget y de Ausubel, teniendo en cuenta la amplia bibliografía existente sobre esas teorías y que el lector podrá consultar. Sí es pretensión mostrar al lector al tanto de los autores que marcaron la formación docente de grado en el período en cuestión, en Argentina.
} 
contribuyan al desarrollo cognitivo y a la autonomía del alumno, ayudándolo a aprender a aprender. ¿Cuándo enseñar? es otro aspecto que el docente debe considerar: los objetivos y contenidos se secuencian siguiendo el ritmo de desarrollo de los alumnos (concepción piagetiana) sin forzar la intervención pedagógica puesto que ésta no puede acelerar ni adelantar los procesos cognitivos de los alumnos, sólo puede maximizarlos para que el alumno logre un aprendizaje significativo. En ese sentido, la incorporación de contenidos está limitada por el nivel de competencias cognitivas de los alumnos.

A pesar de la fuerza que tomó la difusión de la psicología cognitivista, ésta no llegó a consolidar un modelo de formación docente, pero sí llegó a marcar algunas pautas que hasta la actualidad orientan al docente en instancias de organización curricular y planificación de algunos aspectos que orientan su práctica pedagógica.

\section{3 ¿Y en la enseñanza de LE?}

Los avances en investigación de la enseñanza que partieron de Estados Unidos, alcanzaron Europa y llegaron a América del Sur, e impregnaron la ELE en Argentina ya iniciada la década del '80, favorecida por el regreso de la democracia.

El trabajo didáctico comenzaba, de manera paulatina, a romper con la validaciónde modelos lingüísticos americanos, efectuandose elecciones epistemológicas claras en psicología del lenguaje. Es necesario comprender esas elecciones como la construcción de otro modelo de sujeto en las actividades de lenguaje, lo que supone una crítica a la psicología aplicada. Desde este punto de vista, la atención del proceso pedagógico centrada en el alumno (perspectiva piagetiana y de Ausubel), da primacía a la pedagogía y a la necesidad de trabajar principalmente sobre los procesos de adquisición del alumno, sin dejar de considerar la conceptualización del docente como condición ineludible para el aprendizaje de los alumnos. En esa época se buscaba de aclarar el problema pedagógico de las LE para que fuera posible de ser tratado en su especificidad.

La atención centrada en el alumno permitía comprender las habilidades intelectuales que realizaba en el proceso de aprendizaje. La psicología cognitivista daba indicios al docente sobre cómo el sujeto aprendía, permitía reconocer una reestructuración del universo mental del estudiante, lo cual significó cuestionar los hábitos de audición, de articulación 
morfosintácticos, semánticos y culturales. Ocurría una reestructuración casi total de la realidad y de si mismo a través del aprendizaje de una lengua (DALGALIAN y otros, 1981).

¿Por qué ese gran cambio? Para aquella época y en los avances específicos producidos en la DLE, la ELE se iba apartando del reinado del positivismo y de la lingüística para dar atención al sujeto alumno. Esto implicaba reconocerun cambio en la organización del yo, en lo concerniente al docente y a los alumnos, además de reconocer que ese cambio podía ser frecuentemente sentido como una tensión o dificultad para la enseñanza y/o el aprendizaje.

En la balanza entre la motivación y el trabajo intelectual, el alumno iba otorgando su propia impronta en el proceso de aprendizaje: autonomía del alumno (MORALES, 1999). De todos modos, en la ELE se considerabaal estudiante en su individualidad como también en un grupo. Éste último era importante como dispositivo que permitía superar dificultades en el aprendizaje, fortaleciendo el trabajo cooperativo, donde las diversas performances individuales estaban puestas en acción conjuntamente para llevar adelante un plan de acción de aprendizaje basado en la ayuda y no en la sanción del error.

La integración, la evaluación como ayuda, la función del feed-back grupal, la desdramatización del error otorgaron a la ELE un giro de especificidad, de singularidad.

Vemos, así, cómo este enfoque construye un discurso que al colocar al alumno en el centro de la escena pedagógica, daba lugar alconcepto de libertad del alumno para actuar, para hablar (ib).

Es el estudiante quien debe experimentar la necesidad de aprender y de comunicarse, marcada básicamente por sus características socio-culturales. Para esto, la DLE buscaba los medios que podían ayudar en el aprendizaje apoyándose también en un material que fuera lo suficientemente útil y atractivo para los estudiantes. Se daba atención a un saber-hacer en lectura, un saber-hacer comunicativo y un saber-hacer en escritura, siempre teniendo en cuenta la capacidad del alumno de confrontar las nuevas experiencias de aprendizaje con las experiencias que ya poseía.

Si bien el docente es corrido del centro de la escena pedagógica, él va acompañando, guiando al alumno en el aprendizaje. Se reconoce que el docente posee toda una formación que le provee pericia y habilidad en su tarea de enseñanza y en el conocimiento de su objeto de estudio. El balance continuo entre autonomía del alumno y acompañamiento del docente 
también confería a la ELE una especificidad que la distinguía, denominada como "pedagogía no directiva atenuada" (ib) ${ }^{12}$.

Además vale destacar que los avances en la lectura de textos de especialidad, en los estudios de cohesión y coherencia textual, en los actos de habla, enel enfoque comunicativo, fueron generando cambios en la ELE que enlazaban al aprender a aprender del cognitivismo aspectos socio-culturales que marcarían la singularidad de laDLE hasta la actualidad.

\section{$5 \mathrm{El}$ efecto de complejidad}

\subsection{En la investigación de la enseñanza}

Los investigadores procuran alejarse de todo atisbo del positivismo con la finalidad la atender la complejidad de la vida del aula poniendo en evidencia la conjunción de culturas que componen el aula.“...a medida que nos internamos en el mundo de la investigación de la ecología del aula, nos encontramos con una serie de tendencias intelectuales totalmente diferentes" (SHULMAN, ib., p.46).

Esa propuesta de investigación de la enseñanza se caracteriza por tener en cuenta la interacción entre las personas y sus medios en términos de reciprocidad, entender a la enseñanza y al aprendizaje como procesos continuamente interactivos, considerar que el contexto del aula está inmerso en otros contextos que influyen en el aula: el ecosistema del alumno (el aula, el profesor, la escuela, la comunidad), reconocer como fuente de datos a los procesos no observables: pensamientos, actitudes, sentimientos, percepciones de los participantes. Este conjunto de características permite identificar este programa como interpretativo, cualitativo, etnográfico y sociolingüístico.

En este género de investigación, el término culturaes muy importante.Shulman (ib., p.47) apunta las palabras deCliffordGeertz, considerado el portavoz sobre ese término:

El concepto de cultura que sostengo... es fundamentalmente un concepto semiótico. Como creo, con Max Weber, que el hombre es un animal preso en las tramas de

\footnotetext{
${ }^{12}$ Dalgalian propone "une pédagogie non-directiveatténuée" pues considera que siempre en la enseñanza es indispensable una cierta estructuración.
} 
significados que él mismo ha tejido, considero que la cultura consiste en tramas, y que por lo tanto, el análisis de la misma no es una ciencia experimental en busca de leyes sino una ciencia interpretativa en busca de significado. Es una explicación lo que busco, la construcción de expresiones sociales sobre su superficie enigmática.

El docente-investigador que trabaja en esta perspectiva no tiene la finalidad de descubrir leyes como tampoco está preocupado por el valor útil (en el sentido de aplicativo) de los resultados, ya que las concepciones de la enseñanza, de las aulas, delaprendizaje están sustentadas en la idea de que los hechos tienen diferentes significados para los actores ${ }^{13}$ del aula.

Como se indicó antes, en el programa proceso-producto, el profesor era el centro de la vida del aula, la fuente de partida de la enseñanza; en la cognición del alumno y mediación de la enseñanza, ese centro es ocupado por el alumno visto desde una perspectiva sociocognitiva. En el género actual, el centro del escenario es el aula comprendida como ecosistema, por lo tanto como cruce de diversidades culturales; como un medio comunicativo en el cual los hechos de la vida cotidiana forman parte de las interacciones entre profesores y alumnos.

Los investigadores de esta tendencia acostumbran preguntar en qué condiciones culturales podrían ser consideradas algunas prescripciones vigentes en la enseñanza, por ejemplo, las generalizaciones, los resultados donde se indican relaciones causales, la descontextualización de resultados. Para esos investigadores, tales prescripciones podrían ser consideradas incompletas y absolutamente erróneas. La antropología, la sociología y la sociolinguiística, la psicología ecológica, la etnografía son las disciplinas interpretativistasmás influyentes en este género de investigación de la enseñanza y no admiten tales prescipciones.

\section{$5.2 . .$. en la formación docente}

En el actual enfoque, el docente toma un lugar muy particular y complejo. Este es escucha, problematizador, mediador de negociaciones, puente que orienta los pasajes de la cultura hacia las culturas, organizador de la diversidad, fabricante de convergencias...

\footnotetext{
${ }^{13}$ El término "actores" nos sitúa en el enfoque interpretativista del interaccionismo simbólico que caracteriza este programa de investigación.
} 
En ese sentido, consideramos que el docente de LE requiere ser formado en el marco de las tendencias crítico-reflexivas. Sin embargo, en la formación docente no es una tendencia homogénea; pueden distinguirse básicamente tres perspectivas: Una refiere a "la conjunción investigación-acción"; otra, a "la reflexión acerca de las prácticas colectivas desarrolladas durante el proceso de formación"; la tercera, al "reconocimiento de la dimensión política de los actos educativos y consideración de las dimensión gnoseológica de los procesos formativos" (VOGLIOTTI et al., 1989).

La perspectiva que propone "la conjunción investigación-acción" (I-A) es la más conocida en Argentina, aunque poco puesta en práctica en la enseñanza escolar de LE, como también en el ámbito de la formación de profesores. De esta manera, en la carrera de grado, los futuros profesores de LE toman conocimiento de esta perspectiva solamentedesde el punto de vista teóricoal cursar las asignaturas pedagógicas generales.

Como es ampliamente conocido, la finalidad de la I-A está orientada a producir un cambio en la práctica áulica, involucrando docentes y alumnos. La propuesta de trabajo conjunto, la reflexión continua del proceso planificado y puesto en acción, los valores que se comparten y se discuten, son aportes que pueden constituir estrategias de formación del docente que propicien una redefinición del rol docente, de la didáctica constructivista y fortalezcan la gestión de concertación en la construcción de conocimientos (SAGASTIZABAL y PERLO, 2002).

En la perspectiva de la "reflexión acerca de las prácticas colectivas desarrolladas durante el proceso de formación", se consideran las apreciaciones que los docentes sostienen con respecto a sus propias prácticas y a la construcción de los vínculos interpersonales que tienen lugar en la situación pedagógica. En este enfoque se reconocen la dimensión ideológica del conocimiento y la historicidad contextual, o sea las trayectorias individuales de aprendizaje, las matrices, etc. Cobra importancia qué es conocer, cómo se conoce, qué se conoce, quién conoce y con quién se conoce en los procesos formativos ya que se considera que el sujeto que conoce y el objeto de conocimiento se presentan como diferentes pero vinculados en un proceso dialéctico. Aunque esta perspectiva parece muy abstracta y está prácticamente ausente durante la formación de grado, se la encuentra en innúmeras investigaciones que muchos docentes realizan principalmente a nivel de posgrado. 
En la tercera perspectiva, "reconocimiento de la dimensión política de los actos educativos y consideración de las dimensión gnoseológica de los procesos formativos", el conocimiento es concebido como una construcción social y dialéctica en la que se examina/explora explícitamente la naturaleza política de la acción y la reflexión. En ese sentido, el docente se preocupa por: qué es conocer, cómo se conoce, qué se conoce, quién conoce y con quién se conoce en los procesos formativos, para qué se conoce, a favor de qué y de quiénes se conoce, en contra de qué y de quiénes.

Las tres perspectivas indicadas poseen puntos de contacto, aspecto que comparten como por ejemplo: la estrecha relación entre teoría y práctica, se considera que los sujetos no pueden conocer separadamente de su acción, de su vivencia, ni pueden accionar sin reflexión. Desde el punto de vista teórico, estas perspectivas de formación se instrumentalizan en disciplinas de las áreas sociológicas, pedagógico-didácticas y metodológicas. Desde el punto de vista de la práctica, el objeto de conocimiento y el proceso se confunden, no existen separadamente. No se pierde de vista que quien se forma deberá conocer determinados temas, casos, hechos...también se destaca que aprenderá habilidades, aptitudes individuales, grupales, procedimientos interactivos que conllevan a objetivos socialmente compartidos (miembros de los grupos-objetivos de trabajo).

En otras palabras, formar un docente crítico-reflexivo significa que éste reflexione sobre su práctica pedagógica modificándola para alcanzar una re-conceptualización permanente. Perspectiva que en entendemos coherente con la propuesta actual de la ELE.

\section{3 ¿Y en la enseñanza de LE?}

La perspectiva cognitivista que sostenía la importancia del "aprender a aprender" abrió la puerta para la consideración de aspectos sociales que mediaban en el proceso de aprendizaje. La pragmática se perfiló con gran fuerza en el enfoque comunicativo que representa una concepción de lengua como interacción social. Este enfoque, subsidiado por la Pragmática y la Lingüística de texto, imperó en Argentina alrededor de 20 (años '80 y '90), enriquecido por innúmeras investigaciones, discusiones teóricas, experiencias áulicas, etc. creando muchos y diferentes matices en dicho enfoque a lo largo de todo ese tiempo. El concepto de competencia lingüística fue superado por el de competencia comunicativa que 
abraza fuertemente el componente cultural propio de la lengua de estudio, los elementos culturales que posee el estudiante y los que se han de tener en cuenta en el aprendizaje de una LE.

En el marco del abordaje comunicativo se distinguían, inicialmente, dos términos referidos a cultura: multiculturalismo e interculturalismo. De todos modos, en la ELE desde muy larga siempre estuvo presente la preocupación por la relación lengua-cultura:

\begin{abstract}
Quando o alemão era uma das principais línguas que orientava o ensino de língua estrangeira na Europa, já privilegiava o objetivo formativo desse ensino. Segundo Puren (1989), em 1890 já se colocava em discussão a questão do ensino de uma língua pela língua em si mesma. Essa preocupação está claramente expressa no avant-propos do livro-texto "L'allemandetl'Allemagne par lestextes" que fora editado pela editorial Hachette em 1924 (MORALES, 2000, p.140).
\end{abstract}

Como se expresa en la cita, la ELE procuraba enlazar el aprendizaje de la lengua en si misma con la formación del sujeto a través del trabajo con aspectos de la cultura del país donde se hablaba esa lengua, en este caso el alemán y Alemania. Esos aspectos estaban referidos a conocimientos de geografía, la historia, el arte, la literatura. Incluso esa preocupación estaba indicada como estudio de la civilización y no específicamente como cultura. Durante los años de vigencia del enfoque comunicativo, en Argentina se hablaba básicamente de "multiculturalismo" e "interculturalismo". Frente a esos términos, ¿qué ocurre con las culturas?pregunta Zarate $(1983)^{14}$. La autora los presenta metafóricamente como unrendez-vous manqué(un encuentro truncado) y como unmariage(un casamiento)respectivamente.

De todos modos, en la ELE en Argentina, esa diferencia no era crucial pues se trataba de enseñar la cultura propia de la lengua durante el proceso de adquisición de la competencia comunicativa, como se indicó arriba.

El énfasis en los estudios sobre interculturalidad y su integración en la enseñanza de la lengua de forma didáctica empezó a plantearse a inicio del 2000 y actualmente constituye el centro de los discursos de docentes de una LE. Incluso, los manuales para la enseñanza de

\footnotetext{
${ }^{14}$ Considero el texto de Zarate interesante de ser referenciado, pues muestra que la preocupación por el diálogo de culturas, el conocimiento/entendimiento de otra cultura tiene como principal objetivo favorecer una capacidad interpretativa del aluno ligada a la constitución de la identidad: "un savoir-faire interprétatif de l'élève... Céstainsi que l'apprentissage d'une langue étrangèrenepeut, à notre avis, contournercequirelève de l'identité de l'élève" (p. 80). GenevièveZarate es, en la actualidad, una pensadora que continúa realizando interesantes estudios sobre interculturalidad en la enseñanza de lenguas extranjeras.
} 
lenguas indican claramente la perspectiva intercultural queorienta la propuesta con la finalidad de que el alumno pueda conocer y vivir la lengua-cultura en estudio, considerando las relaciones con otras culturas (BARBOSA y CASTRO, 2013).

Para la conceptualización de "interculturalidad" tomamos la concepción de pedagogía intercultural expresada por Abdallah-Preteceille y $\operatorname{Porcher}^{15}$ (1996, p. 8):

L’interculturelreposesurunprincipefortetsimple, déjà dit: l'Autre est à lafoisidentique à moi et différent de moi.S'il manque l'undesdeuxtermes, on se trouveinévitablementprojetéversunenseignement de l'exclusion ou de larelégation. La pédagogieinterculturelle instaure une dynamique, insuffle une viecohérenteàun ensemble par nature disparate. Elle se situedu côté dumouvementcontrel'immobilisme et lespositionsdéfensivesvindicatives, elle fait de l'école à lafoisunlieu d’apprentissage et unlieu de vie.

Como se expresa en la cita, la problemática de la alteridad se coloca en la escena pedagógica a través de la cuestión de lo extraño y extranjero, como también de la percepción de una actualidad percibida como dramática (en sentido de emocionante, conmovedora, teatral), recordando que la alteridad es consubstancial a la educación.

Para esos autores, la alteridad puede ser abordada de la boca para afuera ( $d u$ bout des doigts) quedando sólo en el nivel del discurso sobre el otro, en vez de ser tratada como un encuentro con el otro. El desafío de una pedagogía intercultural consiste en poner al descubierto la cuestión ética del reconocimiento de la alteridad. Además, la enseñanza de la alteridad no es sólo conocer al pasado (la historia) o el presente del otro, sino que implica conocer las maneras como los grupos organizan sus relaciones con el pasado, con el presente según sus intereses y las circunstancias.

También vale la pena destacar que la cultura,al igual que la lengua, constituye un lugar de puesta en escena de uno mismo y los otros. Aunque anclada en la historia, la cultura no se deja encerrar, no se deja atrapar en definiciones cerradas y se abre a diversidad de lecturas e interpretaciones.

El reconocimiento de la cultura en la didáctica de LE se inscribe en una lógica de irrupción de la complejidad y del análisis, sustituyendo la lógica de la justificación y de la explicación culturalista. Enseñar a poner en suspenso nuestras certezas y básicamente

\footnotetext{
${ }^{15}$ Según nuestro punto de vista, este material es muy provechoso para o profesor de L.E., pues aborda diversos aspectos sobre cultura en una perspectiva teórica genérica y también sobre prácticas da alteridad que alcanzan específicamente a la didáctica de las lenguas extranjeras.
} 
lascertezas culturales será un objetivo. Así, la cultura es una lengua, concepto importante para la ELE que pone en evidencia la inadecuada separación lengua y cultura.

\section{CONSIDERACIONES FINALES}

A través del gesto de lectura practicado hemos buscado leer más allá de las teorías que convencionalmente se tratan en la DLE o en las metodologías de ELE. Razón por la cual nos aventuramos a abordar cuestiones teóricas que pocas veces (o casi nunca) se aproximan a la ELE como en este caso ha sido con los géneros de investigación de la enseñanza y las tendencias de formación docente inicial.

El gesto de lectura nos permitió percibir tres grandes efectos de sentido: el tecnocrático, el de especificidad y el de complejidad, que a seguir sintetizamos.

El efecto tecnocrático evidenciado se expresa claramente en la prevalencia de una enseñanza eficaz, del positivismo, la psicología conductista, la vida del aula limitada en relaciones lineales entre docente y alumnos, entre imagen y traducción, imagen y situación, el dominio del profesor de técnicas de enseñanza que aseguraban un buen rendimiento de los alumnos, los ejercicios estructurales que a modo de técnicas dirigían el aprendizaje de los alumnos y no dejaban espacios a actividades de reempleo libre. El dominio de ejercicios repetitivos que realizaban los alumnos para sistematizar los conocimientos, caracterizaba la ELE entre 1950 y 1970 en la formación de profesores de LE, en Argentina.

Es posible, entonces, entender la formación y la práctica docente como una práctica alienada y desprofesionalizante ya que el docente se convierte en mediador y ejecutor de diseños de instrucción, perdiendo autonomía y la posibilidad de tomar de decisiones reflexivas y fundamentadas.

En el efecto de especificidad, hemos podido percibir el camino transitado por la ELE desde una perspectiva estructurada en base a la lingüística americana hacia la búsqueda de aspectos que otorgaban trazos bien delimitados: la concepción de autonomía del alumno, de pedagogía no directiva atenuada, el cuestionamiento de los hábitos de escucha, de los aprendizajes semánticos y morfosintácticos, el balance entretrabajo individual y grupal, la pragmática, son todos aspectos que fueron delineando y consolidando una fisionomía particular de la ELE. En la búsqueda de explicitación de la operacionalidad que se imponía en 
el positivismo, surge esta dimensión de trabajo que da pasos firmes hacia la reflexión didáctica, que no se interesa por mantener en el primer plano a la conquista de cientificidad sino la reivindicación de una especificidad en la ELE.

Podríamos decir que este efecto también es de especificidad porque constituye un puente muy claro entre el efecto tecnocrático y el de complejidad. Puente de tránsito de la simplificación técnica hacia la complejidad.La explicitación de interrogaciones didácticas pone en cuestión la preocupación por el status de cientificidad de la enseñanza tanto en la didáctica de LE como en las disciplinas convergentes, en tanto que ese status se encuentra constantemente parasitado y paralizado en la disputa por la legitimidad.

En el efecto de complejidad percibimos que en el género de investigación de la ecología del aula, como en la tendencia crítico-reflexiva de formación docente y en el enfoque intercultural las orientaciones desembocan en un alejamiento de la LE de esquemas estructurados de enseñanza y de los determinismos culturales.

Aprender a relevar, ver, comprender las modalidades y los sentidos atribuidos a los aspectos culturales en el aula y la comunicación apuntan hacia un hacer cultural. Éste implica reconocer de inicio la propia aula como ecología de culturas, lejos de pensar que la cultura sólo está "allá" en la otra lengua, remolcada por la lingüística.

El enfoque intercultural en la ELE permite claramente establecer vínculos con la concepción ecológica del aula en la cual cada sujeto es una microcultura y el aula es el hábitat que alberga un mestizaje de microculturas, a veces en conflicto, en relación de extrañeza, en búsqueda de simetrías, etc. Ambos están apoyados en aportes teóricos de la socio-lingüística, la etnografía, la sociología, la antropología encarnan los principios teóricos.

El docente asume un lugar relevante en esta propuesta pues, formado en una perspectiva crítico-reflexiva, puede sostener el tono humanista en la enseñanza y moderar una propuesta de ELE sostenida en una epistemología de la complejidad y no de la simplificación. Para finalizar, reproducimos las palabras de Puren (p. 392), ampliamente citado antes y con las cuales coincidimos:...L'histoire de méthodologiesnepeut à la foisqu'êtrelue a partir du présent et être une lecture du présent... ${ }^{16}$

\section{EFEITOS DE SENTIDO NO ENSINO DE LÍNGUA ESTRANGEIRA:}

\footnotetext{
${ }^{16}$ La historia de las metodología sólo puede ser leída a partir del presente y ser una lectura del presente (NdeT)
} 


\title{
UM GESTO DE LEITURA
}

\section{Resumo}

$\mathrm{O}$ artigo visa produzir um relato do ensino de línguas estrangeiras (ELE) na Argentina, entre 1950 e o presente. Para isso, buscou-se destacar a crescente complexidade que permeia esse ensino com o avanço de novas teorias. A partir de uma triangulação teórica entre os programas de pesquisa do ensino, tendências teóricas relativas à formação de graduação de professores e abordagens metodológicas para o ELE, constatam-se três efeitos de sentido: o tecnocrático, da especificidade e da complexidade. Considera-se que a perspectiva epistemológica que reduz a complexidade do ELE a um conjunto de aspectos isolados é abandonada gradualmente, atingindo a atual abordagem pedagógica dominante que ilustra as interações entre a cultura na qual está inserido o ELE e as condições para a superação do marco estreitodessa cultura para aprender com a alteridade.

Palavras-chave: Ensino de Línguas Estrangeiras; Pesquisa do Ensino; Formação de Professores; Efeitos de Sentido

\section{MEANING EFFECTS IN FOREIGN LANGUAGE TEACHING: A READING GESTURE}

\begin{abstract}
This paper attempts to outline the history of foreign language teaching in Argentina since the 1950s. To this end, we sought to highlight the growing complexity in foreign language teaching due to theoretical changes in the field. Using the theoretical triangulation of research programs in the study of teaching, theoretical trends in initial teacher training, and methodological approaches for the teaching of foreign languages, three meaning effects were detected: a technocratic effect, a specificity effect, and a complexity effect. The teaching perspective which consists of reducing complexity to isolated parts seems to be gradually abandoned and the field is predominantly moving to a pedagogical approach which illustrates
\end{abstract}


the interactions between the culture where the teaching takes place and the attempts to move beyond this culture to learn with otherness.

Keywords: Foreign Language Teaching; Research Programs for the Study OfTeaching, Teacher Education; Meaning Effects

\section{REFERENCIAS}

ABDALLAH-PRETCEILLE, Martine \& PORCHER, Louis.Éducationet communication interculturelle.Paris: Pressesuniversitaires, 1996.

BARBOSA, Cibele N., CASTRO, Giselle N.de. Brasil intercultural. Língua e cultura brasileira para estrangeiros.2013. Buenos Aires: Casa do Brasil.

CHIS, Jean-Louis. Revendicationd'autonomie et horizon de scientificité en didactique du français. Revista Langue Française, Paris:Larousse, n.82, p.31-38,mai 1989.

DALGALIAN, Gilbert et al. Pour unnouvelenseignement des langues et une nouvelle formation des enseignants.Paris: CléInternational, 1981.

DUHALDE, Miguel. Pedagogía crítica y formación docente. Paulo Freire.Contribuciones para la pedagogía. Buenos Aires: CLACSO. Disponible en: http://bibliotecavirtual.clacso.org.ar/ar/libros/campus/freire/20Duhal.pdf. Acceso en 20 mayo 2011.

GOMEZ RESTREPPO, Bernardo. Concepto de investigación educativa y tendencias temáticas y metodológicas de la investigación sobre educación en los últimos 30 años. La investigación en Educación. Colombia: Programa de especialización en teoría, métodos y técnicas de la investigación Social, módulo 7, dic. 2002 . Disponible en:http://www.scribd.com/doc/6952950/6-Investigacion-evaluativa. Acceso en 19 sept.2010.

MORALES, Gladys B. A constituição da identidade social do professor de língua estrangeira no curso de formação. 2000. 250f. Tese (Doutorado em Educação)-UFRGS, Porto Alegre.

MORALES, Gladys B. Ensino de língua(s) estrangeira(s) e autonomía do aluno: a inquietude de uma trajetória. Revista Cronía. Año3, vol.3, n 1, p.258-271,1999.

PÊCHEUX, Michel. Ler o arquivo hoje. In: ORLANDI, E. Gestos de leitura. Campinas: Editora da Unicamp,1994.

PUREN, Christian. Des méthodologiesconstituées et de leur mise en question. Revista Le françaisdans le monde. p.36-42, jan. 1995 
PUREN, Christian. Histoire des méthodologies de l'enseignement des langues. Paris: Clé International, 1989.

SAGASTIZABAL, M.A y PERLO,C.L. La investigación-acción como estrategia de cambio en las organizaciones. Bs. As:La Crujía. 2002.

SAUSSURE, Ferdinand. Curso de Lingüística General. Buenos Aires: Losada.1945.

SCHULMAN, Lee. S. Paradigmas y programas de investigación en el estudio de la enseñanza: una perspectiva contemporánea. In:WITTROCK, M. (Org.). La investigación de la enseñanza I. Madrid: Paidós, 1989.

VOGLIOTTI, Ana; MORALES, Gladys. Modelo tecnicistaen formación docente inicial. Revista Jornadas de investigación. Río Cuarto: Facultad de Ciencias Humanas. 1996, p.281285.

VOGLIOTTI, Ana; MACHIAROLA, Viviana; MORALES, Gladys. Formación docente inicial.Contextos, fundamentos, perfiles. Río Cuarto: EFUNARC,1998.

ZARATE, Geneviève. La compétenceculturelle en langueétrangère.Revista Dialogues et cultures. Québec: FIPF, n.25, p.73-80,1983.

Data de recebimento: $18 / 02 / 2015$

Data de aceite: 29/04/2015 